\title{
Go or no-go? Developmental improvements in the efficiency of response inhibition in mid-childhood
}

\author{
Lucy Cragg \& Kate Nation \\ Department of Experimental Psychology, University of Oxford, UK
}
This is the author's post-print of the article. The definitive version was
published in Developmental Science, Volume 11 Issue 6, July 2008. This can be
accessed at http://www3.interscience.wiley.com/cgi-
bin/fulltext/121359037/PDFSTART

\begin{abstract}
This experiment used a modified go/no-go paradigm to investigate the processes by which response inhibition becomes more efficient during mid-childhood. The novel task, which measured trials on which a response was initiated but not completed, was sensitive to developmental changes in response inhibition. The effect of inducing time pressure by narrowing allowable response time was also examined. While increasing time pressure did not reduce the inhibitory demands of the task for either age group, older children (aged 9-to-11 years) were able to inhibit their responses at an earlier stage of movement than younger children (aged 5-to-7 years). This shows that as children get older they become more efficient at controlling their behaviour which drives developmental improvements in response inhibition.
\end{abstract}

Address for correspondence:

Lucy Cragg

School of Psychology

University of Nottingham

University Park

NOTTINGHAM. NG7 2RD, UK

lucy.cragg@nottingham.ac.uk 


\section{Introduction}

Response inhibition is one of the purest forms of inhibition, involving only a choice between an action or a non-action (Rubia et al., 2001). The go/no-go task reflects this and simply requires a response to be made to a frequently occurring 'go' stimulus but withheld when a less frequent 'nogo' stimulus is presented. The relative frequency of go trials compared to no-go trials creates a tendency to respond on every trial (prepotent response) which then has to be inhibited in order to suppress the action. Response inhibition is indexed by the number of no-go trials on which a response is made, which indicates a failure to actively inhibit the response.

Previous studies investigating the development of response inhibition using the go/no-go task have shown mixed results. While some have shown that performance on no-go trials improves with age during childhood (Archibald \& Kerns, 1999; Becker, Isaac, \& Hynd, 1987; Brocki \& Bohlin, 2004; Dowsett \& Livesey, 2000; Levin et al., 1991; Levy, 1980; Livesey \& Morgan, 1991; Luria, 1959) this has not always been found (Johnstone et al., 2007; Jonkman, 2006). In other cases, developmental change is only seen under certain conditions. Espy (1997) showed an improvement with age only when an efficiency score took response speed into account, but not on accuracy alone. Furthermore, Levin et al. (1991) found that a developmental improvement in response inhibition was no longer significant once the number of trials completed by each age group was taken into account. Even for those studies that found a developmental improvement, the age at which response inhibition matures is not clear: Some studies suggest that mature go/no-go performance is achieved around 8 years of age (Becker, Isaac, \& Hynd, 1987), whereas others comparing children up to the age of 12 with adults suggest that response inhibition is still not fully developed in these older children (Booth et al., 2003; Bunge, Dudukovic, Thomason, Vaidya, \& Gabrieli, 2002; Casey et al., 1997). These discrepant findings are difficult to interpret, especially as methodological differences such as the relative frequency of no-go trials (Berwid et al., 2005), the number of go trials preceding a no-go trial (Durston, Thomas, Worden, Yang, \& Casey, 2002) and time pressure (Simpson \& Riggs, 2006) can have a large impact on the pattern of results observed. In addition, differences between age groups are often small. Thus, lack of developmental change may be a consequence of task insensitivity rather than a true reflection of no difference between children of a different age.

While there is at least some evidence that the ability to inhibit a response improves during childhood, the exact process by which this occurs is not clear. Although the standard go/no-go task involves a simple choice between an action and a non-action (Rubia et al., 2001), this does not mean that inhibiting that action is also an all-or-none process. A response to a no-go trial may be initiated but not completed for example. One simple hypothesis is that as children get older, they are able to inhibit their actions at an earlier stage in the execution of the motor response, thereby becoming more efficient at controlling their behaviour. On this view, young children have slow inhibitory processes and therefore are unable to inhibit a response quickly enough to prevent an error being made. As older children have faster inhibitory processes, they are able to inhibit a response before it is completed, thereby avoiding an error. This improves until a response can be inhibited before any movement is made.

The go/no-go task has not been thought of in these terms before. However, another measure of response inhibition, the stop-signal task, is based on a similar principle. Here, participants are presented with stimuli that require a speeded motor response. On some trials a signal occurs at a variable delay after the stimulus, telling the participant to withhold that response. If the stopsignal occurs at a sufficiently short delay the response can be prevented but at longer delays, the stop-signal arrives too late to prevent the response from being executed. Logan and colleagues' horse-race model (Logan \& Cowan, 1984; Logan, Cowan, \& Davis, 1984) proposes a race between two independent processes: selecting and executing a response to the first stimulus, and inhibiting all motor responses. Whichever process is completed first determines whether a response is made. Therefore, the speed of each set of processes is critical. Although not directly observable, inhibition speed can be estimated from other parameters in the task. This is labelled the stop-signal reaction time (SSRT) and quantifies the efficiency of inhibition.

Developmental studies using the stop-signal task provide mixed findings concerning agerelated improvement on the task. While some studies have found a significant decrease in SSRT with age (Bedard et al., 2002; Ridderinkhof, Band, \& Logan, 1999; Williams, Ponesse, Schachar, Logan, \& Tannock, 1999) others have not (Band, van der Molen, Overtoom, \& 
Verbaten, 2000; Jennings, van der Molen, Pelham, Debski, \& Hoza, 1997; Schachar \& Logan, 1990). However, it has been argued that the null results are largely due to a lack of power, suggesting that inhibition processes do in fact become more efficient with age (Band, van der Molen, \& Logan, 2003: Ridderinkhof et al., 1999; Williams et al., 1999). We predict that this is also the case for the go/no-go task: As inhibition processes become faster, responses should be inhibited at an earlier stage of the movement.

The processes involved in the stop-signal task are relatively well understood, whereas those underlying the go/no-go task have not been fully explored. It seems likely that both tasks involve a similar race between a go-process and a stopprocess. There are however some differences between the paradigms. In the stop-signal task, the stop-signal always comes after a signal to respond, so a movement is always started before it has to be inhibited. In the go/no-go task the signal instructs not to make a response in the first place. This can be viewed as a stop-signal task with an SOA of $0 \mathrm{~ms}$, but it has also been argued that additional response selection processes are likely to be involved in the go/no-go task (Johnstone et al., 2007; Rubia et al., 2001).

To test the hypothesis that the stage at which a response is inhibited gets earlier with age, we modified the standard go/no-go paradigm to include a home key that had to be pressed down between trials and on no-go trials, but released to respond using the target key on go trials. This enabled the measurement of partial inhibitions, no-go trials on which the home key was released but the movement was stopped before the target key was pressed. Including the extra partial inhibition measure provided an indication of the stage at which a response was inhibited. Although this does not allow a specific estimation of the covert latency of the inhibition process as in the stop-signal task, it provides a more direct measure of the latency of inhibition that does not rely on an indirect calculation with a number of assumptions, such as that employed to estimate the SSRT. If it is the case that response inhibition becomes more efficient with age then as children get older they should make fewer partial inhibitions and more successful inhibitions, where a response is inhibited before any movement is made.

A go/no-go task with a release key set-up has also been used with older adults (Amieva et al., 2002). Amieva et al. found that the majority of the participants' errors involved releasing the hand from the starting point, but not following through to respond. This suggests that the partial inhibition is a better index of performance on the go/no-go task than the standard measure of the number of completed responses on no-go trials and therefore may be a more sensitive measure for detecting developmental changes.

In addition to the speed of inhibitory processes affecting performance, response speed may also influence go/no-go performance. Simpson and Riggs (2006) argued that if the amount of time given to respond is short, young children may not have enough time to respond, removing the opportunity for inhibitory failure on no-go trials. They suggested that only when accuracy is higher on go trials than on no-go trials can the task be considered to have inhibitory demands as this shows that children understand the task and so do not make errors on go trials, but have particular difficulty inhibiting responses on no-go trials. With a presentation time of $2 \mathrm{~s}$ and a high frequency of go trials this criteria was met, showing that inhibitory demands were high. In contrast, when the presentation time was too short (1s), there was not enough time for the children (aged 3 years) to respond and therefore, no inhibition was observed. Similarly, when presentation time was too long (3s), inhibitory demands were reduced as time pressure was insufficient to make button-pressing prepotent.

To determine whether a restriction on response time affects older children in a similar manner, we manipulated the inter-stimulus interval (ISI). Additionally, by comparing two age-groups of children, we examined whether shortening the response window is more detrimental to younger vs. older children. As children get faster at responding with age, we predicted that reducing the length of the ISI would have a greater effect on the younger children who, given their slower response times, may not have enough time to respond at a shorter ISI.

In summary, this experiment aimed to reveal factors that underpin go/no-go performance. We modified the standard go/no-go paradigm to provide a more sensitive measure of performance as well as to assess the hypothesis that as children get older, they are able to inhibit their responses at an earlier stage during the movement. We also manipulated response time (ISI) to investigate the effects of time pressure across different stages of development. 


\section{Method}

\section{Participants}

Ninety children from primary schools in England participated. Two groups of children were recruited: 5- to 7-year-olds and 9- to 11year-olds, allowing us to examine maximal changes in response inhibition development during mid-childhood. One 5- to 7-year-old was excluded due to an excessive number of anticipatory responses, and a 9- to 11-year-old was excluded due to experimenter error, leaving forty four 5- to 7-year-olds ( $\mathrm{M}=6.55$ years, S.D. $=.58,21$ male) and forty four 9- to 11-yearolds ( $\mathrm{M}=10.57$ years, S.D.=.61, 22 male $)$.

\section{Materials}

A background scene consisting of grass, sky and a football goal was constantly present on the screen (see Appendix). On each trial either a football (go stimulus) or a rugby ball (no-go stimulus) was presented centrally near the bottom of the screen. Rather than simply pressing a key in response to go stimuli and withholding this response for no-go stimuli, participants held down a home key with their index finger between trials and responded to targets using an adjacent key. The 'left-click' mouse button on a laptop was the home key and the 'right-click' mouse button was the target key. A red star sticker was placed on the home key to indicate where to press. If the key was released between trials then a reminder "Press the star!" appeared at the top of the screen. The correct response to a go stimulus was to release the home key and press the adjacent target key with the same finger as fast as possible, whereas the correct response to a no-go stimulus was to continue pressing the home key. This manipulation allowed for a more sensitive measure of task performance, as trials where the participant released the home key but did not press the target key were treated as partial inhibitions that were initiated but successfully inhibited before completion.

\section{Procedure}

To encourage the development of a prepotent response, children first completed two blocks of 30 go trials. Following instruction and 10 practice trials, the first block was presented. The appearance of the target was contingent on the home key being pressed down, after which the football appeared for $200 \mathrm{~ms}$. A variable ISI ensured that the appearance of the target could not be predicted therefore discouraging anticipatory responses. The average length of the ISI was also manipulated with an ISI of $2600-3400 \mathrm{~ms}$ in the first block and $1600-2400 \mathrm{~ms}$ in the second block. Feedback (number of correct responses) was presented after each block.

Following two blocks of go trials, two blocks including no-go stimuli were presented. The children were told that they were going to play a new game that was very similar to the first, but that this time they would sometimes see a rugby ball. It was explained that they should not kick the rugby ball but should keep their finger pressed down on the star button when one appeared. The children completed eight practice trials (including two no-go trials) followed by two blocks of 52 trials. Thirteen trials $(25 \%)$ of each block were no-go trials. Both go and no-go stimuli were presented for $200 \mathrm{~ms}$. To encourage a regular pattern of responding, stimulus presentation was not contingent on the home key being pressed and the targets appeared at fixed regular intervals with an ISI of $3000 \mathrm{~ms}$ in the first block and $2000 \mathrm{~ms}$ in the second block. Feedback (number of correct responses to go trials) was provided at the end of each block.

\section{Results}

Any trial with an RT less than $200 \mathrm{~ms}$ or where the first response was not the release of the home key was classed as an anticipatory trial (7.2\% of trials). To determine if the number of anticipatory responses differed between the two age groups a two-way mixed-measures ANOVA with Age (5-7, 9-11) as a between-subjects factor and Trialtype (go, no-go) as a within-subjects factor was performed. The only significant result was a main effect of Age $F(1,86)=9.59, p<.01$, $\eta^{2}=.100$, which showed that the younger children $(\mathrm{M}=8.06$, S.D. $=8.51)$ made more anticipatory responses than the older children $(\mathrm{M}=3.55$, S.D. $=4.53$ ) on both go and no-go trials. As anticipatory responses did not relate to our hypotheses concerning response inhibition they were not considered further.

Responses to go trials were coded as either hits (the home key was lifted and the target key was pressed) or misses (the home remained pressed or the home key was lifted but the response key not pressed). Responses to no-go trials were coded as either successful inhibitions (the home remained pressed), partial inhibitions (the home key was lifted but the response key not pressed) or failed inhibitions (the home key was lifted and the target key was pressed). Each response type was calculated as a percentage of 
the total number of go or no-go trials within a block. For the RT analyses, RTs greater than 2.5 SDs above the participant's mean for each measure (release time: stimulus-release; movement time: release-press) were excluded (release time: $0.02 \%$ excluded; movement time: $1.18 \%$ excluded).

We present three sets of analyses on these responses. The first two analyses treated the partial inhibition measure in different ways to assess the advantage of using this measure in the go/no-go paradigm. Our first analysis classed partial inhibitions on no-go trials as correct. This is similar to standard go/no-go paradigms in which any no-go trial on which a response is not completed is treated as correct. To determine if including the partial inhibition measure provided a more sensitive measure of performance, our second set of analyses re-classified partial inhibitions to no-go stimuli as errors. Finally, our third analysis examined the number of each type of response for no-go trials to directly assess the hypothesis that as children get older they can inhibit a response at an earlier stage during the movement.

\section{(i) Standard analyses}

For go trials hits were treated as correct and misses treated as incorrect. Successful and partial inhibitions were treated as correct for no-go trials, whereas failed inhibitions were treated as incorrect. The number of correct responses were calculated as a percentage of the overall number of go or no-go trials for each ISI and entered into a three-way ANOVA with Age (5-7, 9-11) as a between-subjects factor, and ISI (2000ms, $3000 \mathrm{~ms}$ ) and Trialtype (go, no-go) as withinsubject factors. There was a main effect of Trialtype, $\mathrm{F}(1,86)=38.4, \mathrm{p}<.001, \eta^{2}=.308$, reflecting more correct responses for go trials $(\mathrm{M}=85.2$, S.D.=10.2) than for no-go trials $(\mathrm{M}=74.4$, S.D.=20.2) showing that the task created inhibitory demands. Consistent with the findings of Simpson and Riggs (2006) there were more correct responses when the ISI was longer (3000ms: $M=81.5$, S.D.=13.9; 2000ms: $M=78.2$, S.D.=15.4) as shown by a main effect of ISI, $\mathrm{F}(1,86)=9.66, \mathrm{p}<.01, \eta^{2}=.101$. Overall, 9-11-yearolds $(M=82.7$, S.D.=13.8) made more correct responses than 5-7-year-olds $(M=76.9$, S.D.=13.3) as shown by a main effect of Age, $F(1,58)=3.97$, $\mathrm{p}=.05, \eta^{2}=.044$. There was no Age $x$ Trialtype interaction showing that performance on both go and no-go trials improved with age.

RT analyses were performed for hits on go trials only. RTs for partial inhibitions are analysed in the next section while RTs for failed inhibitions could not be analysed due to the small number of these responses at each ISI. Two 2way ANOVAs were used, one with release time as the dependent variable and the other with movement time as the dependent variable. In both analyses, ISI $(2000 \mathrm{~ms}, 3000 \mathrm{~ms})$ was a withinsubject factor and Age (5-7-, 9-11) a betweensubjects factor. Overall 9-11-year-olds were faster to respond than 5-7-year-olds as shown by a main effect of Age for both release time $(\mathrm{F}(1$, 86) $=57.4, \quad \mathrm{p}<.001, \quad \eta^{2}=.040 ; \quad$ 5-7-year-olds: $\mathrm{M}=450$, S.D. $=89.2$; 9-11-year-olds: $\mathrm{M}=330$, S.D.=57.0) and movement time: $(\mathrm{F}(1,86)=31.1$, $\mathrm{p}<.001, \quad \eta^{2}=.265 ; \quad$ 5-7-year-olds: $\mathrm{M}=187$, S.D.=68.0; 9-11-year-olds: $M=125$, S.D.=30.3). The home key was released more quickly in the $2000 \mathrm{~ms}$ ISI condition $(\mathrm{F}(1,86)=5.72, \mathrm{p}<.05$, $\eta^{2}=.062 ; 2000 \mathrm{~ms}: \mathrm{M}=386$, S.D. $=97.8 ; 3000 \mathrm{~ms}$ : $\mathrm{M}=394$, S.D.=97.0) but there was no effect of ISI on movement time $(\mathrm{F}(1,86)<1)$. There was no Age $x$ ISI interaction for either measure (release time; $\mathrm{F}(1,86)<1$; movement time: $\mathrm{F}(1,86)=2.09)$ showing that manipulating the amount of time to respond on go trials affected both age groups in the same way.

In summary, accuracy was higher on go trials than no-go trials, and when the ISI was longer. All children were slower to release the home key with a longer ISI, suggestive of a speed-accuracy trade-off. 9-11-year-olds were faster than 5-7year-olds on go trials and more accurate on both go and no-go trials.

\section{(ii) Analysis including partial inhibitions}

Hits were treated as correct and misses treated as incorrect for go trials. Only successful inhibitions were treated as correct for no-go trials, whereas failed inhibitions and partial inhibitions were treated as incorrect. Thus, some no-go trials which were classed as correct in the previous analyses (i.e. if the if the home key had been released but the target key was not pressed) were now classed as partial inhibitions and scored as incorrect.

A three-way ANOVA was performed on the percentage of correct responses, with Age (5-7, 9$11)$ as a between-subjects factor, and ISI $(2000 \mathrm{~ms}$, $3000 \mathrm{~ms}$ ) and Trialtype (go, no-go) as withinsubject factors. There was a main effect of Trialtype, $\mathrm{F}(1,86)=569, \mathrm{p}<.001, \quad \eta^{2}=.869$, reflecting more correct responses for go $(\mathrm{M}=85.0$, S.D.=10.7) than for no-go trials $(M=36.7$, S.D.=22.9). While there was no main effect of ISI $(\mathrm{F}(1,86)<1)$ there was an interaction between ISI and Trialtype, $F(1,86)=18.2, p<.001, \eta^{2}=.175$. 
Table 1. Means (S.D.) and F values for one-way ANOVAs comparing children who did or did not make more than 5 partial inhibitions on no-go trials for each ISI condition

\begin{tabular}{|c|c|c|c|}
\hline & $\begin{array}{l}\text { More than } 5 \text { partial } \\
\text { inhibitions in each ISI } \\
\text { condition }\end{array}$ & $\begin{array}{l}\text { Less than } 5 \text { partial } \\
\text { inhibitions in each ISI } \\
\text { condition }\end{array}$ & $\begin{array}{r}F \\
\text { value }\end{array}$ \\
\hline$\underline{5-7-y r-o l d s}$ & $\mathrm{~N}=16$ & $\mathrm{~N}=28$ & \\
\hline Age (years) & $6.66(0.67)$ & $6.49(0.52)$ & $<1$ \\
\hline WASI vocabulary & $23.2(6.32)$ & $21.9(6.97)$ & $<1$ \\
\hline WASI matrices & $12.0(6.55)$ & $10.7(6.85)$ & $<1$ \\
\hline SNAP $^{1}$ inattention score & $0.19(0.28)$ & $0.35(0.47)$ & 1.49 \\
\hline Anticipation trials & $6.69(8.15)$ & $8.84(8.76)$ & $<1$ \\
\hline Go release $\mathrm{RT}$ & $444.9(54.8)$ & $453.5(105)$ & $<1$ \\
\hline Go release RT variability & $129.4(42.9)$ & $130.8(44.7)$ & $<1$ \\
\hline PI release $\mathrm{RT}$ & $415.5(52.4)$ & $420.8(110)$ & $<1$ \\
\hline 9-11-yr-olds & $\mathrm{N}=13$ & $\mathrm{~N}=31$ & \\
\hline Age (years) & $10.7(0.64)$ & $10.5(0.60)$ & $<1$ \\
\hline WASI vocabulary & $38.5(6.65)$ & $38.7(8.77)$ & $<1$ \\
\hline WASI matrices & $17.5(5.74)$ & $18.8(5.11)$ & $<1$ \\
\hline SNAP $^{1}$ inattention score & $0.64(0.77)$ & $0.35(0.52)$ & 2.14 \\
\hline Anticipation trials & $3.65(4.04)$ & $3.52(4.78)$ & $<1$ \\
\hline Go release $\mathrm{RT}$ & $301.2(26.3)$ & $341.3(62.4)$ & $4.96^{*}$ \\
\hline Go release RT variability & $67.7(11.3)$ & $85.8(45.0)$ & 2.02 \\
\hline PI release RT & $286.1(31.1)$ & $309.3(50.5)$ & 2.31 \\
\hline
\end{tabular}

Notes: ${ }^{*} \mathrm{p}<.05 ;{ }^{1}$ Swanson, Nolan and Pelham (SNAP) rating scale. Includes items from the DSM-IV criteria for ADHD and the Conners Index Questionnaire. PI = partial inhibition

Accuracy improved on no-go trials when the ISI was shorter (3000ms: $\mathrm{M}=34.3$, S.D. $=23.1$; 2000ms: $M=39.1$, S.D. $=25.8)$, but decreased on go trials (3000ms: $M=87.2$, S.D. $=10.6 ; 2000 \mathrm{~ms}$ : $\mathrm{M}=82.8$, S.D.=14.2), suggesting that there was not enough time for the children to respond on go trials when the ISI was reduced. A marginally significant interaction between Age and ISI, $\mathrm{F}(1,86)=3.82, \mathrm{p}=.054, \eta^{2}=.043$, indicated that the younger children were particularly affected by this increase in time pressure $(3000 \mathrm{~ms}: \mathrm{M}=56.8$, S.D. $=13.8 ; 2000 \mathrm{~ms}: \mathrm{M}=54.3$, S.D.=16.2), In contrast, the older children were more accurate with a shorter ISI $(3000 \mathrm{~ms}: \mathrm{M}=64.7$, S.D. $=14.3$; 2000ms: $M=67.6$, S.D.=16.9). Overall, older children (M=66.2, S.D.=14.8) were more accurate than younger children $(\mathrm{M}=55.5, \mathrm{~S} . \mathrm{D} .=13.7), \mathrm{F}(1$, 86) $=10.8, p=.001, \eta^{2}=.111$. Again, there was no Age $x$ Trialtype interaction $(\mathrm{F}(1,86)=1.75$, ns $)$ indicating that younger children made fewer correct responses on go as well as no-go trials. A significant correlation between correct go trials and correct no-go trials, controlling for age, showed that children who made more correct go responses were also more likely to inhibit responses on no-go trials, $r=.506, \mathrm{p}<.001$.
As there were relatively few partial inhibitions made in each condition, RT data were only analysed for the 16 younger and 13 older children who made at least five partial inhibitions in each ISI condition so that a reliable RT measure was obtained. Before describing the results of this analysis, it is important to determine whether the two groups of children (those who made at least five partial inhibitions and those that did not) differed on key variables such as age, IQ and aspects of task performance, such as speed, RT variability and number of anticipation trials. Relevant data are presented in Table 1. We analysed group differences for the two age groups separately as we hypothesised that there would be different reasons for older and younger children making partial inhibitions. For younger children, partial inhibitions may be seen in children with more mature performance, reflecting a move away from failed inhibitions. In contrast, the older children who make more partial responses may be those with more impulsive performance. Overall, we found no differences between the younger children who made more or less than five partial inhibitions. However, the older children who made more than five partial responses were faster to release the home key on go trials, suggesting 
they may be more impulsive. This tentative finding should be investigated further in future experiments.

Release time RTs were entered into a threeway ANOVA with Age (5-7, 9-11) as a betweensubjects factor, and ISI (2000ms, 3000ms) and Response (hit, partial inhibition) as within-subject factors. There was a main effect of Age, $\mathrm{F}(1$, 27) $=75.0, \mathrm{p}<.001, \eta^{2}=.735$, as overall 9-11-yearolds were faster than 5-7-year-olds $(\mathrm{M}=294$, S.D. $=26.7$ vs. $M=430$, S.D. $=51.4)$. There was also a main effect of ISI, $\mathrm{F}(1,27)=8.02, \mathrm{p}=.01$, $\eta^{2}=.229$ as responses were faster when the ISI was shorter (3000ms: $M=378$, S.D. $=83.4 ; 2000 \mathrm{ms:}$ $\mathrm{M}=360$, S.D.=80.9). The home key was released more quickly on partial inhibition trials $(\mathrm{M}=357$, S.D.=78.6) than on correct hits on go trials $(\mathrm{M}=380$, S.D. $=84.8)$ as shown by a main effect of Trialtype, $\mathrm{F}(1,27)=19.5, \mathrm{p}<.001, \eta^{2}=.419$. This suggests that faster responses were less likely to be inhibited. There was no Age Group x Trialtype interaction $F(1,27)=1.99$ suggesting that the greater number of partial inhibitions in younger children was not simply due to the fact that they were responding more quickly on these trials.

While the results including partial inhibitions mirror those seen in the standard analyses, it is interesting to note that considering partial inhibitions reduced the number of correct responses on no-go trials from $77 \%$ to $43 \%$ in the 9-11-year-olds, and from $72 \%$ to $31 \%$ in the $5-7$ year-olds. This demonstrates that all children often inhibited their response after the movement had been initiated but before its completion. Thus including the partial response measure provided a more accurate assessment of task performance than simply measuring completed responses on no-go trials. Considering the partial inhibition measure also revealed age differences between the ISI conditions that were not present in the standard analyses. Furthermore, for those children making more than five partial inhibitions in each ISI condition, these partial inhibitions were faster than hits on go trials, consistent with the idea that fast responses are initiated before inhibitory processes are completed.

\section{(iii) Responses to no-go trials}

If response inhibition becomes more efficient with age, younger children should make more partial inhibitions on no-go trials than older children, who should be more likely to fully inhibit their response. To test this hypothesis, we compared the number of each type of response (successful inhibition, partial inhibition, failed inhibition) made by each age group on no-go trials (Figure 1).

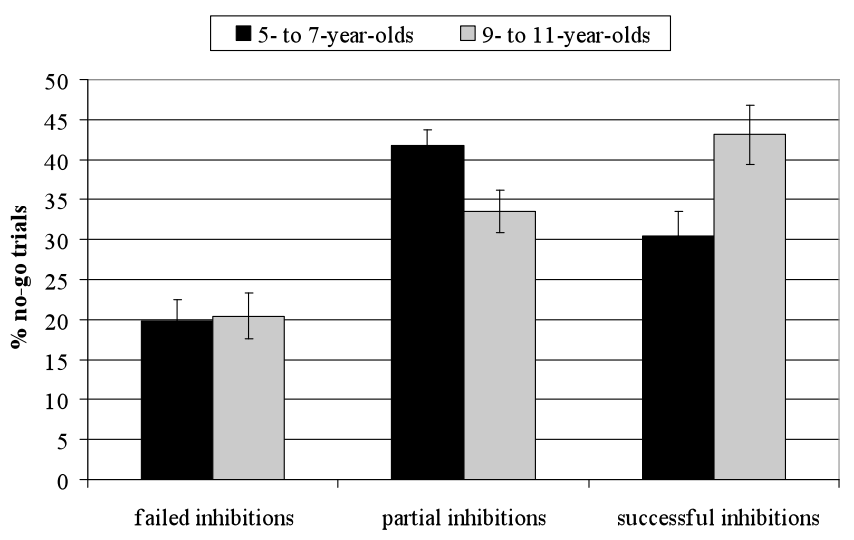

Figure 1. Mean (S.E.) percentage of each response type made on no-go trials by 5-to 7-year-olds and 9-to 11-year-olds.

A three-way mixed measures ANOVA was performed with Age (5-7, 9-11) as a betweensubjects factor, and ISI (2000ms, $3000 \mathrm{~ms})$ and Response (successful inhibition, partial inhibition, failed inhibition) as within-subject factors. Main effects of Age, $F(1,86)=10.6, p<.01, \eta^{2}=.110$, and Response, $\mathrm{F}(1.81,156)=16.4, \mathrm{p}<.001, \eta^{2}=.160$ were qualified by a Response $\mathrm{x}$ Age Group interaction, $\mathrm{F}(1.81,156)=4.63, \mathrm{p}<.05, \eta^{2}=.051$. This showed that the younger children $(M=41.8$, S.D.=13.3) made more partial inhibitions than the older children $(\mathrm{M}=33.5$, S.D.=17.6), whereas the older children made more successful inhibitions than the younger children (5-7: $\mathrm{M}=30.5$, S.D.=19.6; 9-11: $\mathrm{M}=43.1$, S.D.=24.4), confirming our predictions. In contrast to some previous findings (Archibald \& Kerns, 1999; Brocki \& Bohlin, 2004) there was no difference in the number of failed inhibitions made by the two age groups (5-7-year-olds: $\mathrm{M}=19.8, \mathrm{~S} . \mathrm{D} .=17.4 ;$ 9-11year-olds: $M=20.5$, S.D.=19.0). A main effect of ISI, $\mathrm{F}(1,86)=12.1, \mathrm{p}=.001, \eta^{2}=.123$, was qualified by an interaction between ISI and Response, $\mathrm{F}(1.89, \quad 162)=7.75, \mathrm{p}=.001, \quad \eta^{2}=.083$, which showed that the ISI manipulation affected the response types in different ways. There was no effect of ISI on the number of failed inhibitions. Importantly however, partial inhibitions were not caused by insufficient time to complete the response as there were more successful inhibitions with a shorter ISI (3000ms: $\mathrm{M}=34.5$, S.D. $=23.0$; 2000ms: $M=39.1$, S.D.=25.9) and more partial inhibitions with an ISI of $3000 \mathrm{~ms} \quad(\mathrm{M}=41.4$, S.D. $=18.3$ vs. $\mathrm{M}=33.8$, S.D.=18.8).

To add further support to our hypothesis that response inhibition becomes more efficient as children get older, correlations between age 
(entered as a continuous variable) and performance were carried out. Consistent with our predictions, the number of partial inhibitions made decreased with increasing age $r=-.240$, $\mathrm{p}<.05$, whereas the number of successful inhibitions increased with age, $r=.295, \mathrm{p}<.01$.

\section{Discussion}

This experiment compared the performance of two age groups of children on a version of the go/no-go paradigm modified to capture the stage at which a movement is inhibited. This allowed us to investigate how changes in the efficiency of response inhibition underlie developmental improvement on the go/no-go task. As expected, our standard analyses showed that accuracy was higher on go trials than on no-go trials, confirming that the task is tapping into inhibitory processes. The children understood what they were supposed to do and tended to respond correctly to go stimuli but had greater difficulty inhibiting responses to no-go stimuli. There was an overall improvement with age demonstrating developments in response activation processes on go trials as well as response inhibition on no-go trials.

Further analyses investigated the nature of this developmental change in response inhibition. Taking partial inhibitions into account revealed the same pattern of results as standard analyses. Importantly however, this more sensitive response measure demonstrated that successful inhibition on no-go trials occurred on only $43 \%$ and $31 \%$ of trials for 9-11-year-olds and 5-7-year-olds respectively, compared to the inflated figures of $77 \%$ and $72 \%$ suggested by the standard analyses ${ }^{1}$. This shows that many responses by both age groups were partial inhibitions, classed as successful inhibitions in the standard paradigm. Thus, our modified task demonstrates that on many trials children were inhibiting responses after they had been initiated but before they were completed.

Although we showed a developmental improvement in accuracy on no-go trials, further analyses showed no difference in the number of failed inhibitions made by the two age groups. While this is in contrast to some developmental studies which have shown a reduction in failed

${ }^{1}$ It should be noted that the use of a releasepress response rather than a simple press response means that our standard analyses are not directly comparable to typical go/no-go paradigms used in other studies. inhibitions in a similar age group (Archibald \& Kerns, 1999; Brocki \& Bohlin, 2004), it is consistent with others in showing that failed inhibitions are not a sensitive measure of the development of response inhibition (Johnstone et al., 2007; Jonkman, 2006). Go/no-go performance is heavily influenced by task parameters such as the relative frequency of no-go trials (Berwid et al., 2005) the number of go trials preceding a no-go trial (Durston et al., 2002) and time pressure (Simpson \& Riggs, 2006) and therefore differences in the stimuli, response demands, or even the type of analyses used, may have contributed to discrepancies between studies.

In contrast, our new partial inhibition measure was sensitive to developmental change. It revealed that 5- to 7-year-olds made more partial inhibitions on no-go trials than 9- to 11-year-olds, who in turn inhibited more no-go responses before any movement was made. Although both age groups were able to halt some responses before completion, the older children were more likely to inhibit responses at an earlier stage in the movement, before it had been initiated, whereas more of the younger children were inhibiting their responses at a later stage, after it had been initiated. In line with our prediction, the efficiency of response inhibition improves during mid-childhood. This complements findings from the stop-signal paradigm where developmental improvements in the speed of inhibition have also been found (Bedard et al., 2002; Ridderinkhof et al., 1999; Williams et al., 1999). Together, these results strengthen the argument that as children get older they are able to inhibit a response at an earlier stage in its execution.

For those children who made more than five partial inhibitions in each ISI condition, release times for partial inhibitions on no-go trials were generally faster than hits on go trials. This may reflect the fact that these faster responses are initiated before inhibitory processes are completed, consistent with Logan and Cowan's (1984) horse-race model. A further prediction following from this is that failed inhibitions would be faster still than partial inhibitions. We were unable to test this however, due to the small number of failed inhibitions.

It is important to note that older children were more accurate than younger children on go trials as well as no-go trials, consistent with earlier studies (Archibald \& Kerns, 1999; Berwid et al., 2005; Brocki \& Bohlin, 2004; Levin et al., 1991). This demonstrates developmental change in other factors, as well as response inhibition. Potentially, misses on go trials may reflect a lapse 
in monitoring processes or sustained attention (Berwid et al., 2005). While there may be different processes underlying errors on go and no-go trials, a significant correlation between performance on go and no-go trials suggests that there are some factors, such as stimulus recognition, which contribute to successful performance on both types of trial.

Performance on go trials may also have been affected by the amount of time given to respond on each trial. Consistent with this suggestion, Simpson and Riggs (2006) found that manipulating the time to respond affected the balance of errors on go versus no-go trials in preschoolers, and therefore whether or not the task could be considered to have inhibitory demands. Manipulating the ISI allowed us to investigate the influence of availability of response time in our experiment. We anticipated that a shorter response time would have a greater effect on the younger children, as they would be slower to respond and therefore a shorter response window would result in greater time pressure in this age group. There was a trend towards a decrease in the younger children's performance in the $2000 \mathrm{~ms}$ condition, compared to a slight increase in performance in the older children. However, this did not affect the balance of go and no-go errors in the two groups. Overall the pattern of results for both age groups with an ISI of either $2000 \mathrm{~ms}$ or $3000 \mathrm{~ms}$ was very similar; accuracy was higher on go trials than on no-go trials in both conditions, showing that the task was posing inhibitory demands for all children at both ISIs. This contrasts with Simpson and Riggs' finding that reducing the presentation time by $1 \mathrm{~s}$ resulted in lower accuracy on go trials than on no-go trials in pre-schoolers. This suggests that while manipulations of time pressure may affect preschooler's performance on the no-go task, small changes in ISI do not affect patterns of performance in school-age children. However, it may be that shorter ISIs than those used in this study would have a more detrimental effect on school-aged children's performance.

In conclusion, this study used a novel version of the go/no-go paradigm to test the hypothesis that children's performance on the task improves as they become more efficient at inhibiting their responses. The younger children were found to inhibit their movements at a later stage in response execution than the older children, supporting this hypothesis. Further research is needed in order to conduct a more fine-grained analysis of these developmental changes.

\section{Acknowledgements}

This work was supported by the Biotechnology and Biological Sciences Research Council. We are grateful to the staff and pupils of the participating schools for their help and cooperation and we thank Gaia Scerif for helpful comments on a previous draft of the manuscript.

\section{References}

Amieva, H., Lafont, S., Auriacombe, S., Le Carret, N., Dartigues, J-F., Orgogozo, J-M., et al. (2002). Inhibitory breakdown and dementia of the Alzheimer type: A general phenomenon? Journal of Clinical and Experimental Neuropsychology, 24(4), 503516.

Archibald, S. J., \& Kerns, K. A. (1999). Identification and description of new tests of executive functioning in children. Child Neuropsychology, 5(2), 115-129.

Band, G. P. H., van der Molen, M. W., \& Logan, G. D. (2003). Horse-race model simulations of the stop-signal procedure. Acta Psychologica, $112,105-142$.

Band, G. P. H., van der Molen, M. W., Overtoom, C. C. E., \& Verbaten, M. N. (2000). The ability to activate and inhibit speeded responses: Separate developmental trends. Journal of Experimental Child Psychology, 75, 263-290.

Becker, M. G., Isaac, W., \& Hynd, G. W. (1987). Neuropsychological development of nonverbal behaviors attributed to "frontal lobe" functioning. Developmental Neuropsychology, 3, 275-298.

Bedard, A., Nichols, S., Barbosa, J. A., Schachar, R., Logan, G. D., \& Tannock, R. (2002). The development of selective inhibitory control across the lifespan. Developmental Neuropsychology, 21(1), 93-111.

Berwid, O. G., Curko Kera, E. A., Marks, D. J., Santra, A., Bender, H. A., \& Halperin, J. M. (2005). Sustained attention and response inhibition in young children at risk for Attention Deficit/Hyperactivity Disorder. Journal of Child Psychology and Psychiatry, 46(11), 1219-1229.

Booth, J. R., Burman, D. D., Meyer, J. R., Lei, Z., Trommer, B. L., Davenport, N. D., et al. (2003). Neural development of selective attention and response inhibition. NeuroImage, 20, 737-751.

Brocki, K. C., \& Bohlin, G. (2004). Executive functions in children aged 6 to 13: A 
dimensional and developmental study. Developmental Neuropsychology, 26(2), 571593.

Bunge, S. A., Dudukovic, N. M., Thomason, M. E., Vaidya, C. J., \& Gabrieli, J. D. E. (2002). Immature frontal lobe contributions to cognitive control in children: Evidence from fMRI. Neuron, 33, 301-311.

Casey, B. J., Trainor, R. J., Orendi, J. L., Schubert, A. B., Nystrom, L. E., Giedd, J. N., et al. (1997). A developmental functional MRI study of prefrontal activation during performance of a go-nogo task. Journal of Cognitive Neuroscience, 9, 835-847.

Dowsett, S. M., \& Livesey, D. J. (2000). The development of inhibitory control in preschool children: effects of "executive skills" training. Developmental Psychobiology, 36(2), 161174.

Durston, S., Thomas, K. M., Worden, M. S., Yang, Y., \& Casey, B. J. (2002). The effect of preceding context on inhibition: an eventrelated fMRI study. NeuroImage, 16, 449-453.

Espy, K. (1997). The Shape School: Assessing executive function in preschool children. Developmental Neuropsychology, 13(4), 495499

Jennings, J. R., van der Molen, M. W., Pelham, W., Debski, K. B., \& Hoza, B. (1997). Inhibition in boys with attention deficit hyperactivity disorder as indexed by heart rate change. Developmental Psychology, 33, 308318.

Johnstone, S. J., Dimoska, A., Smith, J. L., Barry, R. J., Pleffer, C. B., Chiswick, D., et al. (2007). The development of stop-signal and go/nogo response inhibition in children aged 7-12 years: Performance and event-related potential indices. International Journal of Psychophysiology, 63(1), 25-38.

Jonkman, L. M. (2006). The development of preparation, conflict monitoring and inhibition from early childhood to young adulthood; a go/nogo ERP study. Brain Research, 1097(1), 181-193.

Levin, H. S., Culhane, K. A., Hartmann, ' Evankovich, K., Mattson, A. J., Harward, F et al. (1991). Developmental changes performance on tests of purported frontal lol functioning. Developmental Neuropsycholog 7(3), 377-395.

Levy, F. (1980). The development of sustain attention (vigilance) and inhibition in childre some normative data. Journal of Chi Psychology and Psychiatry, 21, 77-84.
Livesey, D. J., \& Morgan, G. A. (1991). The development of response inhibition in 4- and 5-year-old children. Australian Journal of Psychology, 43(3), 133-137.

Logan, G. D., \& Cowan, W. B. (1984). On the ability to inhibit thought and action: a theory of an act of control. Psychological Review, 91, 295-327.

Logan, G. D., Cowan, W. B., \& Davis, K. A. (1984). On the ability to inhibit thought and action: A model and a method. Journal of Experimental Psychology: Human Perception and Performance, 10, 276-291.

Luria, A. R. (1959). Experimental study of the higher nervous activity of the abnormal child. Journal of mental deficiency research, 3, 1-22.

Ridderinkhof, K. R., Band, G. P. H., \& Logan, G. D. (1999). A study of adaptive behavior: Effects of age and irrelevant information on the ability to inhibit one's actions. Acta Psychologica, 101(2-3), 315-337

Rubia, K., Russell, T., Overmeyer, S., Brammer, M. J., Bullmore, E. T., Sharma, T., et al. (2001). Mapping motor inhibition: conjunctive brain activations across different versions of go/no-go and stop tasks. NeuroImage, 13, 250-261.

Schachar, R., \& Logan, G. D. (1990). Impulsivity and inhibitory control in normal development and childhood psychopathology. Developmental Psychology, 26(5), 710-720.

Simpson, A., \& Riggs, K. J. (2006). Conditions under which children experience inhibitory difficulty with a "button-press" go/no-go task. Journal of Experimental Child Psychology, 94(1), 18-26.

Williams, B. R., Ponesse, J. S., Schachar, R., Logan, G. D., \& Tannock, R. (1999). Development of inhibitory control across the lifespan. Developmental Psychology, 35(1), 205-213.

Appendix: Examples of (a) go and (b) no-go stimuli (actual stimuli in colour)
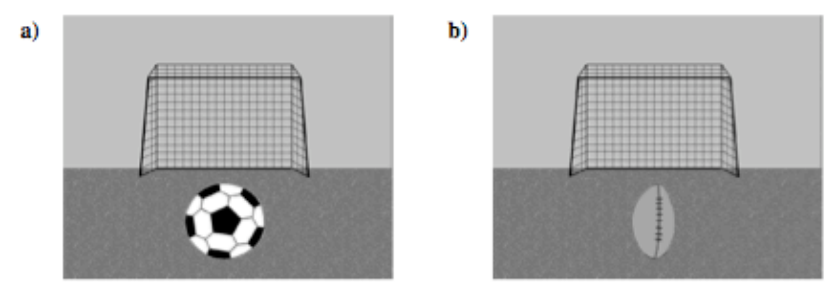